\title{
"DE REDUCTIONE ARTIUM AD THEOLOGIAM" DE SÃO BOAVENTURA (IV).
}

\author{
(Conclusão) .
}

\section{NACHMAN FALBEL}

Do Departamento de História da Faculdade de Filosofia, Letras e Ciências Humanas da Universidade de São Paulo.

\section{CONCLUSÃO.}

A tentativa de classificação das ciências e o seu papel relativo ao conhecimento das Escrituras Sagradas ou a divinitas começou com os primeiros doutores da Igreja. Talvez, porque fôsse inevitável desde o início do cristianismo, institucionalisado, uma relação íntima entre a

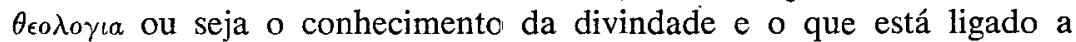

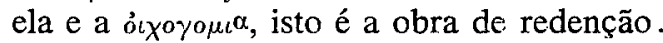

Apesar da posição de Santo Agostinho em ver as ciências humanas como mera expressão da vaidade (137) (nesse caso a própria obra do Santo não escaparia a esta observação, pois ela em grande parte é fruto destas ciências), sabemos que foi grande a preocupação de conhecer o papel que assumia e o lugar que ocupava a teologia no conjunto do saber. Portanto, uma classificação das ciências (138) implicava numa posição direta ou indiretamente assumida perante a teologia (139).

E a posição de Santo Agostinho, que fundamentou o pensamento no ocidente medieval durante muitos séculos até a revolução aristoté-

(137). - De doctrina Christiana, II cap. 42.

(138). - Veja-se alguns exemplos marcantes mencionados por Ghellinck, (J. de), Le mouvement théologique du XIIe siècle, Museum Lessianum, 1948, p. 91 .

(139) . - Para o histórico do têrmo e suas acepçóes o artigo "Théologie" no Dict. Th. Cath., vol. XV, de M. J. Congar. 
lico - tomista do século XIII, era a de que as ciências humanas são "introdutorias", são "exempla" ao conhecimento mais elevado.

Porém o desenvolvimento da gramática, ou a ciência dos nomes, principalmente com o impulso dado pela escola palaciana no reinado de Carlos Magno permite uma aprofundisação no significado do texto da sacra pagina (140). Com o nominalismo de Abelardo, que ainda é uma crítica ao nomen, associado ao método diáletico será dado o salto seguinte para a fundamentação da Teologia como futura ciência . São Boaventura herdará êste passado que se incorpora no curriculum da Faculdade de Teologia da Universidade de Paris.

Ao se formar os studia generalia na ordem Franciscana, (Bologna, Oxford 1229 e Paris 1231) o estudo da teologia se incorporou a Ordem (141).

Já na Faculdade de Teologia se sedimenta o método pedagógico da doctrina sacra exposta pelos magistri. A questio metódica é a forma para a teologia tomar pouco a pouco consciência dos problemas a superar como scientia (142).

A Ordem seguiu a tradição agostiniana - platonisante de acôrdo com os padrões reinantes nos diversos studia, mas não podemos deixar de observar que o significado da expressão é um pouco vago para definir o seu conteúdo especificamente relacionado a cada um dos pensadores ou teólogos da ordem. Daí, os autores que estudam o pensamento medieval divergirem nas denominações e na nomenclatura da corrente ou correntes agostinianas e entre as expressões encontramos a de "agostinísmo franciscano", "agostinísmo aristotélico", agostinísmo avicenisante" (E. Gilson), "agostinísmo avicebronisante" (M. Wulf). $\mathrm{E}$ van Steenberghen (143) chega a definir

la philosophie de Saint Bonaventure est un aristotélisme éclectique et néoplatonisant, mis au service d'une théologie augustinienne.

Mas podemos considerar a obra espiritual de São Boaventura como um passo adiante na afirmação da teologia como ciência cuja elaboração racionalista final far-se-á com São Tomás de Aquino. São

(140). - Chenu (M. D.), La Théologie comme science au XIIIe siècle, Lib. Philos. J. Vtin, Paris, 1957, p. 18.

(141). - Glorieux (P.), Répertoire des maitres en Théologie de Paris au XIIle siècle, J. Vrin, Paris, 1933, vol. II, p. 9.

(142). - Chenu (M. D.), La Théologie au douzième siècle, Lib. Philos. J. Vrin, Paris, 1957, pp. 337-341 .

(143). - Van Steenberghen, op. cit., p. 464. 
Boaventura define o trabalho do teólogo como a elaboração racional da fé e isto é facilitado ao se adotar o livro das Sentenças de Pedro Lombardo como base de ensino, anteriormente já introduzido no currículo por Alexandre de Hales, pai da escola franciscana. Há aqui uma básica diferença de método, servindo o simbólico e a narrativa ao estudo das Escrituras (Scriptura Sacra) que alimenta a doctrina sacra iluminada pela fé. Mas a teologia deve ir além do texto e elaborar os dados da interpretação num inteligível racional, como que considerando a verdade revelada capaz de passar pela prova racional.

Assim sendo o conhecimento teológico implica numa inquirição e numa elaboração racional. Este é o significado da diferenciação feita por São Boaventura do credibile ut credibile (fé) e do credibile ut intelligibile (teologia) (144) que São Tomás distinguirá nìtidamente mais tarde. Isto desloca, como bem observa Chenu 145), o eixo onde giram as formas literarias associadas à teologia, exigindo com isto outras técnicas de explicação. E o que São Boaventura denomina determinatio distrahens, isto é, um deslocamento do eixo primitivo para um outro, ou seja uma subalternação em outros têrmos, apesar de tudo resta sempre no caráter independente da ciência teológica uma subalternação a uma ciência mais elevada, ou seja a de Deus (146).

No fundo o próprio conceito da subalternação explicará a redução de tôdas as ciências a teologia .

Do dito se infere que o conceito de teologia elaborado por São Boaventura não é diferente basicamente a da escola franciscana desde seu fundador, ou seja, Alexandre de Hales. O texto extraido da Summa dêste último reflete perfeitamente o modêlo da conceituação empregada na definição da teologia pela escola (147). São Boaven-

(144). - Comm. in IV lib. Sent. I, Proem. qu. II, 4.

(145). - Chenu (M. D.), La Théologie comme science au XIIle siècle, J. Vrin, Paris, 1957, p. 56.

(146). - Comm. in IV lib. Sent. I, Proem. qu. II, 4: 'Quoniam igitur sacra Scriptura est de credibili ut credibili, hic est de credibili at facto intelligibili, et haec determinatio distrahit - "nam quod credimus debemus auctoritati, et quod intelligimus rationi" - hinc est, quod sicut alius modus certitudinis est in scientia superiori et inferiori, ita alius modus certitudinis est in sacra Scriptura et alius in hoc libro, et ideo alius modus procedenti. Et sicut scientia subalternata, ubi deficit, redit ad certitudinem scientia subalternandis, quae maior est; sic etiam, cum magistro deficit certitudo rationis, recurrit ad auctoritatis certitudinem sacrae Scripturae, quae excedit omnem certitudinem rationis".

(147). - Alexandre de Hales, Summa Theologica, Introd. art. I, cit. em Chenu, op. cit., pp. 38-39. "Notandum quod est scientia causae et scientia causati. Scientia vero causae causarum est sui gratia; scientia vero causatorum sive sint causae sive sint causae sive effectus tantum, non est sui gratia, quia referuntur et dependent a causa causarum. Hinc est quod theologia, quae est scientia de Deo, qui est causarum, sui gratia est. Nomen ergo scientiae appro- 
tura não fugirá apesar de tudo, a êste madêlo no opúsculo que foi objeto dêste trabalho. A teologia é uma ciência ordenada a perfeição do homen e de suas potências da alma, o entendimento e a vontade. A perfeição do entendimento não tem caráter de fim, senão de meio, enquanto que é ordenada a mover a vontade a ação em ordem a possessão de Deus. As verdades reveladas que levam a mover a alma humana por meio do entendimento, provocam uma ativação dos afetos da. vontade. Dêste modo ditas verdades causam na alma certo gôsto ou sabor divino, de onde deriva a palavra sabedoria (sapientia) com que comumente se designa esta ciência. Teologia não é scientia mas é sapientia. Daí que, ainda recnhecendo a função de ilustrar o entendimento, se assinale o fim último da Teologia como ciência que leva a ação externa ou interna. E daí também concluir-se que a teologia apresenta mais afinidade com o grupo das ciências práticas (sed principalius debet dici practica) e não teóricas (148).

Essa concepção, que foi dominante na escola franciscana, em última instância afirma mais uma vez que o saber teologal emana da luz da fé, ainda que de um modo próprio ela como ciência adota princípios, axiomas, conclusões idênticamente a tôda ciência. Só que na teologia os artigos da fé fazem o papel das suppositiones na ciência.

Em resumo o mundo ideal agostiniano - platônico ou seja, a realidade inteligível, é o verdadeiro objeto da sapientia; o mundo sensível, as realidades temporais, a da "ciência". A teologia no esquema de São Boaventura se volta a primeira sem desconhecer a segunda. Trata-se mais de uma diferenciação de graus determinada pela própria natureza do conhecimento humano, onde um não anula o outro, mas servem de escalas até a ascensão final, isto é, a Luz Eterna que

priatur scientiae causatorum, nomen vero sapientiae scientiae causae causarum. Unde et ipse Philosophus dicit quod philosophia prima, quae est sui gratia et de causa causarum, debet dici sapientia. Simili ratione doctrina theologiae, quae trancendit omnes alias scientias, debet dici sapientia. Praeterea, notandum quod est scientia perficiens cognitionem secundum veritatem; est etiam scientia movens affectionem vel bonitatem. Prima est ut cognitio secundum visum, et ideo debet dici scientia absoluta; secunda, ut cognitio secundum gustum, et ideo debet dici sapientia a sapore affectionis, secundum quod dicitur: Sapientia secundum suum nomen est. Theologia igitur quae perficit animam secundum affectionem, movendo ad bonum per principia timoris et amoris, proprie et principaliter est sapientia. Prima philosophia, quae est theologia philosophorum, que est de causa causatorum, sed ut perficiens cognitionem secundum viam artis et ratiocinationis minus proprie dicitur sapientia. Ceterae vero scientiae, quae sunt de causis consequentibus et causatis, non debent dici sapientiae, sed scientiae".

(148). - Comm. in IV lib. Sent 1, Proem, qu. III, 3: "Item omnis scientia, quae est ut boni fiamus, est practica". 
deu origem a tôdas as luzes (a exterior, a inferior, a interior e a superior) e para a qual devem retornar.

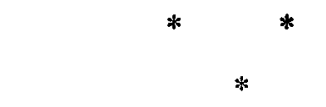

\section{LISTA DE ABREVIATURAS.}

AFH - Archivum Franciscanum Historicum .

AHDLMA - Archives d"histoire doctrinale et litteraire du moyen âge.

ALKG - Archiv für Litteratur und Kirchengeschichte.

Dict. Th. Cath. - Dictionnaire de Theólogie Catholique.

EHR - The English Historical Review.

$\mathrm{FF}$ - La France Franciscaine.

FS - Franziskanische Studien.

\section{BIBLIOGRAFIA.}

FONTES.

AGOSTINUS (S.), De natura boni, T. III (B. A. C.), 1951.

- Contra Academicos, T. III, (B. A. C.), 1951.

- De quantitate animae, T. III, (B. A. C.), 1951.

De immortalitate animae, T. III, (B. A. C.), 1951.

De doctrina cliristiana, MPL.

De utilitate credendi, T. IV, (B. A. C.), 1948.

- De civitate Dei, T. XVI-XVII, (B. A. C.), 1964.

BONAVENTURA (S), Obras, VI tomos, B. A. C., Madrid.

- Opera Omnia, 10 vol., Ad Claras Aquas (Quaracchi), 1882-1902.

- Commentarius in IV libros Sententiarum (Opera Theologica Selecta), Ad Claras Aquas, 1934, 1938, 1941, 1949.

CHARTULARIUM UNIVERSITATIS PARISIENSIS, ed. Denifle-Chatelian, Paris, 1899, 4 vols., Impression anastatique, Bruxelles, Culture et $\mathrm{Ci}$ vilisation, 1964 .

FRANCISCO DE ASSIS (São), Escritos completos y biografias de su época, B. A. C., Madrid, 1956.

HUGO DE S. VICTOR, Eruditione Didascalica, MPL, 176.

MATHEUS DE PARIS, Chronica Majora, MGH, Script., T. XXVIII.

ROGERI BACONIS, Compendium stidii philosophiae, extratos MGH, Script.; T. XXVIII.

SALIMBENE, Chronica, MGH, Ecript., T. XXXII. 
AUTORES MODERNOS.

AMOROS O. F. M., (P. Leon), La teologia como ciencia practica en la escuela franciscana en los tiempos que precedem a Escoto, in "AHDLMA", T. IX, 1934, pp. 261-303.

CHENU (M.-D.), La première diffusion du thomisme à Oxford. Klapwell et ses "Notes" sur les Sentences, in "AHDLMA", T. III, 1928, pp. 185-200.

L_La theologie comme science au XIIle siècle, in "AHDLMA", T. II, 1927, pp. 31-72.

- Grammaire et theólogie aux XIIe et XIIIe siècles, in "AHDLMA", T. X, 1935 e 1936, pp. 5-28.

— La theólogie comme science au XIIIe siècle, Lib. Philos, J. Vrin, Paris, 1957.

_La theólogie au douzième siècle, Lib. Philos. J. Vrin, Paris, 1957.

DICKSON (CH.), Le cardinal Robert de Courzon. Sa vie., in "AHDLMA", T. IX, 1934, pp. 55-142.

DENIFLE (H.), Die Constitutionem des Prediger-Ordens vom Jahre 1228, in "ALKG", T. I, pp. 165-227.

D'IRSAY (STEPHEN), Histoire des universités françaises et etrangères des origines a nos jours, ed. Auguste Picard, Paris, 1933, 2 vol.

DICTIONNAIRE D'HISTOIRE ET GEOGRAPHIE ECCLESIASTIQUES.

EHRLE (F.), Beitrage zur Geschichte der mittelalterlichen Scholastik, in "ALKG", T. V, pp. 603-635.

FELDER (H.), Los ideales de San Francisco de Assis, ed. Descleé, Buenos Aires, 1948 .

FELDER; (H.), Geschichte der wissenschaftlichen studien im Franziskaner Orden, Freiburg, 1904; também ed. ital. Storia degli studi scientifici nell'Ordine Francescano dalla sua fondazione fino a circa la meta del sec. XIII, Siena, 1911.

FRAILE, (G.), O. P., Historia de la Filosofia. Judaísmo. Cristianismo. Islam, T. II, B. A. C., Madrid, 1966.

GENICOT (L.), Le XIIIe siècle européen, Nouvelle Clio, Paris, 1968.

GHELLINCK (J. de), Le mouvement theólogique du Xlle siècle, Museum Lessianun, 1948.

GILSON (E.), L'esprit de la philosophie médievale, Lib. Philos. J. Vrin, Paris, 1944.

_- La philosophie de S. Bonaventura, Lib. Philos. J. Vrin, Paris, 1943.

Les sources gréco-árabes de l'augustinisme avicennisant, in "AHDLMA", T. IV, 1929-1930, pp. 5-149.

Roger Marstons un cas d'augustinisme avicennisant, in "AHDLMA", T. VIII, 1933, pp. 37-42.

GLORIEUX (P.), Répertoire des maitres en théologie de Paris au XIIIe siècle, Lib. Philos, J. Vrin, Paris, 1933, 2 vol.

GUTTMANN (J.), Philosophies of Judaism, Anchor Book, N. York, 1966. 
HUSIK (I.), A history of medieval jewish philosophy, Meridian Books, Philadelphia, 1960.

KNOWLES (D.), The religious orders in England, Cambridge, 1950.

- Some aspects of the career of Archbishop Pecham, in "EHR", vol. 57, jan. 1942, pp. 1-18; 178-201.

LANDRY (B.), L'idée de chretienté chez les scolastiques du XIIIe siècle, Pres. Univ. de France, Paris, 1929.

LITTLE (A. G.), The Franciscan School at Oxford in the.13th century, in AFH, XIX, 1926, pp. 813-74.

LONGPRE, O. F. M. (E.), L'école franciscaine, in "FF", T. VI, 1923, pp. 108-134.

MUNK (S.), Mélanges de philosophie juive et arabe, Lib. Philos. J. Vrin, Paris, 1955.

PElAYO (M. M.), Historia de los heterodoxos, B. A. C., Madrid, 1965, 2 vol.

ROHMER (J.), La theorie de l'abstraction dans l'école franciscaine d'Alexandre de Hales e Jean Peckham, in "AHDLMA", T. III, 1928, pp. 105-184.

_- Sur la doctrine franciscaine des deux faces de lâme, in "AHDLMA", T. II, 1927, pp. 73-77.

RASHDALL (H.), The universities of Europe in the middle ages, Oxford University Press, 1951, 3 vol.

THOUZELlieR (CH.), La place du De Periculis de Guillaume de Saint-Amour dans les polemiques universitaires du XIIIe siècle, in "RH", clvi, 1927, pp. 69-83.

VAN STEENBERGHEN (F.), Siger de Brabant, Les Philosophes Belges, T. XIII, Louvain, 1942, 2 vol.

VOGT (B.), O. F. M., Der Ursprung un d die entwicklung der Franziskanerschule, in "FS", 1922, pp. 137-158.

VANDEN WYNGAERT (A.), Querelles du clergé séculier et des ordres mendiants (XIIle siècle), in "FF", T. V, 1922, pp. 257-281; 369-397.

WADDING (L.), Annales Minorum. Tomos I e II, Roma, 1732.

WULF (M. de), Histoire de la philosophie médievale, 1929.

\section{APENDICE 1 .}

AS CIENCIAS NO ESQUEMA DE SÃO BOAVENTURA SEGUNDO O OPUSCULO "DE REDUCTIONE ARTIUM AD THEOLOGIAM".

IV GRUPOS.

1. - LUZ EXTERIOR = ARTES MECÂNICAS.

2. - LUZ INFERIOR $=$ CONHECIMENTO SENSITIVO.

3. - LUZ INTERIOR $=$ CONHECIMENTO FILOSÓFICO. 
4. - LUZ SUPERIOR = ESCRITURAS SAGRADAS (GRAÇA).

1. - ARTES MECÂNICAS SE RAMIFICAM EM 7 GRUPOS:

a). - TEXTIL (LANIFICIUM).

b). - FABRIL (ARMATURA).

c). - AGRICULTURA.

d). - CAÇA (VENATIO).

e). - NAVEGAÇÃO (NAVIGATIO).

f). - MEDICINA.

g). - TEATRO (THEATRICA).

(constituem as 7 artes mecânicas no Didascalicon de Hugo de S. Victor).

2. - CONHECIMENTO SENSITIVO SE SUBDIVIDE EM 5 FORMAS SEGUNDO OS SENTIDOS CORPORAIS:

a). - VISTA = LUZ PURA QUE DISTINGUE AS COISAS CORPORAIS (QUINTA ESSENCIA).

b). - AUDIÇÃO = LUZ QUE SE MESCLA COM O $A R$.

c). - OLFATO = LUZ QUE SE MESCLA COM O VAPOR (FOGO).

d). - PALADAR = LUZ QUE SE MESCLA COM A AGUA.

e). - TATO = LUZ QUE SE MESCLA COM A TERRA.

3. - CONHECIMENTO FILOSÓFICO QUE SE DIVIDE:

a). - RACIONAL $=$ LÓGICA = DISCURSIVA.

1). 一 GRAMÁTICA.

2). - LÓGICA.

3). -- RETÓRICA.

b). NATURAL $=$ FÍSICA.

1). - FISICA (PROD.).

2). - MATEMÁTICA.

3). - METAFISICA.

c). - MORAL $=$ PRÁTICA $=$ MOTIVA.

1). - MONÁSTICA.

2). - ECONÔMICA (OU DOMESTICA).

3). - POLÍtiCA.

4. - ESCRITURAS SAGRADAS, POSSUI UM SENTIDO LITERAL E TRES SENTIDOS ESPIRITUAIS:

a). - ALEGÓRICO = (FE) (que nos ensina o que temos de crêr da divindade) DOUTORES - SANTO AGOSTINHO (SANTO ANSELMO).

b) . - MORAL $=($ COSTUME) (que nos ensina o bem viver) $P R E$ DICADORES — SÃO GREGÓRIO (SÃO BERNARDO).

c). - ANAGÓGICO = (FIM DE AMBAS) (que nos mostra o caminho de nossa união com Deus) - CONTEMPLATIVOS - 
SÃO DIONÍSIO (RICARDO DE SÃO VICTOR). (POREM HUGO DE SÃO VICTOR SE DESTACOU NAS TRES).

TÔDA LUZ OU ILUMINAÇÃO SE ORIENTA A DEUS, DE ONDE TEVE SUA ORIGEM. TODAS ELAS SE REDUZEM A TEOLOGIA PORQUE SE CONOTAM:

a). - a geração e encarnação do Verbo;

b). - o modo de vida bem ordenada visando a eternidade;

c). - a união de Deus e a alma.

\section{APENDICE II.}

TÁBUA CRONOLÓGICA (V. STEENBERGHEN vol. I. pp. 746-750). (Para um roteiro dos principais acontecimentos ligados à Universidade de Paris).

1198 - 8 janeiro - Eleição de Inocêncio III (16 de julho 1216).

1198 - Morte de Averroes.

1200 - Privilégio de Filipe-Augusto em favor das escolas de Paris.

1204 - Tomada de Constantinopla pelos Cruzados.

1204 a 1210 c. - Roberto de Courçon mestre de Paris.

1206 a 1210 c. - Prevostin de Cremone, chanceler da Universidade de Paris.

1210 - Concílio de Paris.

1215 - agôsto - Estatuto de R. de Courçon.

1216 - 18 julho - Eleição de Honório III (18 março 1227).

1217 - 18 agôsto -- Michel Scotus publica sua tradução da : Esfera de Alpertragius.

1217 - 12 setembro - Chegada dos Predicadores em Paris.

1218 - Filipe se torna chanceler da Universidade de Paris.

1219 - Chegada dos Menores em Paris.

1220 - Jordão da Saxônia entre os Predicantes.

1220 - c. Summa Aurea de Guilherme de Auxerre.

1220 - 21 outubro - Michel Scotus se encontra em Bologna.

1222 a 1237 - Jordão da Saxônia mestre geral da Ordem ('1237).

1226 - Se torna mestre em Paris (?).

1227 - Michel Scotus publica sua tradução do De Caele.

1227 - 19 março — Eleição de Gregório IX ('22 agôsto 1241).

1228 - 10 abril - Guilherme de Auvergne bispo de Paris ('1249).

1228 - 7 julho - Carta de Gregório IX aos teólogos de Paris.

1228 - Primeiras Constituições dos Predicantes.

1229 - 18 março - Sermão de Eudes de Chateauroux em Paris.

1229 - 27 março - Início da greve escolar em Paris.

1229 - 2 abril - Fim da guerra albigense.

1229 - Fundação da Universidade de Toulouse. 
1229 a 1230 - Rolando de Cremona O. P. mestre em Paris.

1230 a 1235 - Hugo de Saint-Cher O. P. mestre em Paris.

1230 - 22 setembro - João de Saint-Gilles se torna Predicante.

1230 - c. - Penetração de Averroes em Paris.

1230 - c. - João Pagus mestre de artes em Paris.

1231 - 13 abril - Carta de Gregório IX à Universidade de Paris.

1231 - 20 abril - Carta de Gregório IX ao abade de Saint-Vitor.

1231 - 23 abril - Carta de Gregório IX sôbre a revisão de Aristóteles.

1231 - Alexandre de Hales se torna frade Menor (?).

1231 - 3 novembro - Morte de Guilherme de Auxerre.

1233 a 1242 - Guerric de Saint-Quentin O. P. mestre em Paris.

1234 - c. - Summa theologica de Rolando de Cremona.

1235 - c. - Rogério Bacon chega a Paris (?).

1236 - antes - Morte de Michel Scotus.

1236 - 23 dezembro - Morte do chanceler Filipe.

1236 a 1247 - Guilherme de Saint-Amour mestre de artes em Paris.

1238 ou 1241 - João de la Rochelle sucede a Alexandre de Hales.

1240 - c. - Guilherme Shyreswood mestre de artes em Paris.

1240 - c. - Pedro de Espanha mestre de artes em Paris.

1240 - c. - De ortu et div. scientiarum de R. Kilwardby.

1240 - junho - Herman o Dalmata traduziu a Ética de Averroes.

1240 - Alberto o Grande chega a Paris (?).

1240 - c. - Summa de creaturis de Alberto Magno.

1240 - c. - Nascimento de Siger de Brabant.

1240 a 1243 c. - Roberto Grosseteste traduz a Etica de Aristóteles.

1240 a 1243 c. - Rogério Bacon mestre de artes em Paris (?).

1242 a 1248 - Alberto o Grande mestre em Paris (?).

1243 - Corpus quase completo do Averroes latino.

1243 - 25 junho — Eleição de Inocêncio IV ('7 dez. 1254).

1243 - Boaventura entra nos Menores.

1245 - Morte de Alexandre de Hales e de João de la Rochelle.

1245 - 22 setembro - Aristóteles interditado em Toulouse.

1245 a 1248 - Eudes Rigaud mestre em Paris.

1245 a 1250 c. - Alberto o Grande publica suas Sentencias.

1246 - Pedro de Espanha em Sienna.

1247 - c. - Rogério Bacon deixa Paris (?).

1247 - 21 dezembro - Condenação de João de Brescain.

1248 - Alberto o grande deixa Paris para ir a Colônia.

1249 - 30 março - Morte de Guilherme de Auvergne.

1249 ou 1250 - Roberto de Sorbon mestre de Paris.

1250 - c. - Lambert de Auxerre mestre de artes em Paris.

1250 - c. - Herman o Dalmata traduziu a Retórica de Aristóteles.

1250 e seguintes - Boaventura comenta is Sentenças em Paris.

1251 - Rogério Bacon volta a Paris. 
1252 antes de 16 fev. - Estatuto da Nação inglêsa da Faculdade de Artes.

1252 perto do outuno - Tomás chega em Paris.

1252 ou 1255 - Gerard de Abeville mestre em Paris.

1252 - c. - Morte de João de Garlande.

1253 - c. - Boaventura é recebido como mestre em Paris.

1253 - Fundação do Colégio de Sorbonne em Paris.

1253 - Morte de R. Grosseteste.

1253 a 1255 - Tomás comenta as Sentenças em Paris.

1254 - Nícolas de Paris é indicado em Paris.

125412 dezembro - Eleição de Alexandre IV ('1261).

1254 a 1263 - Humberto de Romans mestre geral dos Predicadores.

1255 - 19 março - Estatuto da Faculdade de Artes.

1255 a 1260 - Siger chega em Paris.

1255 - De periculis de Guilherme de Saint-Amour.

1256 - início - Tomás é recebido como mestre em Paris (?).

1255 - c. - Tomás escreve De ente et essentia e De principiis naturae.

1256 - Pentecostes - Capítulo geral dos Predicadores em Paris.

1256 - Alberto refuta Averroes em Anagni.

1256 - Herman o Dálmata traduz a Poética de Averroes.

1256 - João de Sicca Villa escreve De principiis naturae.

1256 a 1259 - Tomás disputa $D e$ veritate.

1256 a 1259 - Tomás disputa as Quolibets VII a XI (?).

1256 a 1270 - Principais parafrases de Alberto o Grande (?).

1257 ou mais tarde - Rogério Bacon torna-se franciscano.

1257 - 2 fevereiro - Boaventura é eleito ministro geral da Ordem.

1258 a 1264 - Tomás escreve a Summa contra os Gentios.

1259 - 19 junho - Capítulo geral dos Predicadores em Valenciennes.

1259 - prox. outono - Tomás deixa Paris para ir a Itália .

1259 a 1264 - Pedro de Tarentaise mestre em Paris.

1260 - Guilherme consegue em Nicéia a trad. dos Meteoros de Alexandre de Afrodisia.

1260 - 23 dezembro - Guilherme consegue em Tebas a trad. do De part. animalium de Aristóteles.

1260 a 1265 c. - Nascimento do aristotelísmo heterodoxo.

1261 - 29 agôsto - Eleição de Urbano IV ('1264).

1261 a 1264 - Tomás e Guitherme (?) na Curia em Orvieto.

1263 - 19 janeiro - Urbano IV renova as interdições de 1231.

1263 - Nícolas de Paris é nomeado em um documento.

1263 - Etienne Tempier mestre e chanceler.

1263 - c. - Gilles de Roma torna-se bacharel em artes (?).

1264 a 1268 - Tomás comenta o De anima, II-III.

1265 - 5 fevereiro - Eleição de Clemente IV ('268).

1265 - maio-junho - Nascimento de Dante Alighieri.

1265 a 1267 - Tomás se encontra em Roma (Saint-Sabine). 
1265 a 1268 - Tomás disputa De potentia Dei (?).

1265 a 1268 - Bacon escreve o Opus Maius (dedicado a Clemente IV).

1265 a 1270 - Primeiros escritos de Siger.

1266 - Guilherme consegue a trad. das Categorias de Simplicius.

1266 - 27 agôsto - Siger é citado em uri decreto do legado — Simon de Brion.

1266 a 1268 - Tomás escreve a Io $^{\text {pars. }}$.

1266 a 1272 - Tomás comenta a Metafísica.

1267 - quaresma - Boaventura prega em Paris De decem praeceptis.

1267 - fim - Tomás passa de Roma a Viterbo (Curia).

1268 - fev. a maio - Boaventura prega em Paris "De septem donis Sp. Scti".

1268 ou mais tarde - Guilherme (?) traduz o De anima de Temistius (?) .

1268 - 18 maio - Guilherme consegue a trad. de Element. Theol. de Procles.

1268 - 7 outubro - Etienne Tempier bispo de Paris ('1279).

1268 - outuno - Tomás disputa em Viterbo De spirit. creaturis.

1268 - c. 20 nov. - Tomás deixa Viterbo para Paris (?).

1268 - 29 nov. - Morte de Clemente IV. Sede vacante até 1271.

1268 - $17 \mathrm{dez}$. - Guilherme consegue a trad. do De anima de J. Philipon.

1269 - jan. - Tomás chega a Paris (?).

1269 - maio - Tomás assiste ao capítulo dominicano em Paris.

1269 - 1 ' semestre - Tomás disputa o De anima.

1269 - c. Páscoa - Quodlibet I de Tomás.

1269 - c. Natal - Quodlibet II de Tomás.

1269 a 1271 - Tomás comenta a Fisica.

1269 a 1271 - Tomás disputa De malo.

1269 a 1271 - João Pecham ensina em Paris.

1269 a 1272 - Henri Bate, Pedro de Auvergne, God. de Fontaines, Gilles de Roma, entendent Tomás em Paris.

1269 a 1273 - Tomás comenta o Livro das Causas e o De anima, I.

1269 a 1273 - Siger comenta o Trat. dos Meteoros.

1270 - c. Páscoa - Quodlibet III de Tomás.

1270 - c. Páscoa - Carta de Gilles a Alberto (Mandonnet, S. P. 105 (?)

1270 - 20 julho - Sermão de Tomás em Paris (Mand. S. P. 109) (?).

1270 - Tomás escreve De unitate intellectus.

1270 - $10 \mathrm{dez}$. - Primeira condenação do aristotelísmo heterodoxo.

1270 - c. Natal - Quodlibet XII de Tomás (?).

1271 - Tomás escreve a Ia IIae.

1271 - c. - Tomás escreve De aeternitate mundi (?).

1271 - c. Páscoa - Quodlibet IV de Tomás (?) .

$1271-1^{9}$ set. - Eleição de Gregório X ('1276).

1271 - c. Natal - Quodlibet V de Tomás.

1271 - Guilherme consegue em Viterbo o De caelo de Simplicius. 
1271 a 1272 - Tomás disputa De virtutibus.

1271 a 1272 - Tomás escreve a IIa IIae.

1271 a 1272 - Siger escreve Impossibilia, De necessitate, De aeternitate.

1272 - c. Páscoa - Quodlibet VI de Tomás.

1272 - 25 março - Eleição e cissão na Faculdade de Artes.

1272 - $1^{\circ}$ abril - Estatuto da Faculdade de Artes.

1272 - Tomás disputa De unione Verbi.

1272 após o 29 maio - Tomás deixa Paris para a Itália.

1272 - 13 set. - Morte de Guilherme de Saint-Amour.

1272 - 8 nov. - Morte de Gerard de Abbeville.

1272 - outono - Tomás ensina em Nápoles.

1272 - c. - Siger comenta a Metafísica.

1272 ou 1273 - Siger escreve De an. int., Q. natur. (?).

1272 e 1273 - Tomás escreve a IIIa pars, De substantiis separatis, De caelo,

De generatione, De meteoris.

1272 a $1275-$ De piurificatione intell. de Gilles de Roma.

1273 - 9 abril-28 maio - Boaventura prega em Paris In Hexaemeron.

1273 - 25 abril - Sermão de Boaventura em Paris.

1273 - c. - Siger comenta a Fisica I-IV (?).

1273 a 1275 - Siger expõe as Qu. mórales et natur. (?).

1274 - 7 março - Morte de Tomás em Fossanuova.

1274 - 2 maio - Carta da Faculdade de Artes ao capítulo dominicano de Lyon.

1275 - 7 maio - Fim da cissão na Faculdade de Artes; Pedro de Auvergne reitor.

1274 - 7 maio - Abertura do Concílio de Lyon.

1274 - 15 julho - Morte de Boaventura em Lyon.

1275 - 5 dez. - Ordenação da Faculdade de Artes.

1275 a 1277 - Siger comenta o De anima, a Fisica VIII, a Parva naturalia.

1276 - 10 jan. - Morte de Gregório X.

1276 - 21 janeiro - Eleição de Inocêncio $V$ (Pedro de Tarentaise, '22 junho).

1276 - 11 julho - Eleição de Adriano V ('18 agôsto).

1276 - 2 setembro - Decreto contra o ensino secreto.

1276 - 8 setembro - Eleição de João XXI (Pedro de Espanha, '1277).

1276 - 6 dezembro - Decreto do legado Simão de Brion.

1277 - 18 janeiro - Carta de João XXI a Tempier.

1277 - 7 março - Segunda condenação dc aristotelísmo.

1277 - 18 março - Proibição de Oxford por R. Kilwardby.

1277 - 28 abril - Nova carta de João XXI a Tempier.

1277 - Viagem de Alberto a Paris.

1277 - 20 maio — Morte de João XXI.

1277 - 23 novembro - Citação de Siger e Bernier pelo inquisidor.

1277 - 25 novembro - Eleição de Nícolas III ('1280).

1278 - 9 abril - Guilherme arcebispo de Corinto. 
1279 - 28 janeiro - João Pecham arcebispo de Cantuária.

1279 - 3 setembro - Morte de Etienne Tempier bispo de Paris.

1280 - 22 agôsto - Morte de Nicolau III.

1280 - 15 novembro - Morte de Alberto em Colônia.

1281 - 22 fevereiro - Eleição de Martim IV (Simão de Brion '1285).

1281 a 1284 - Morte de Siger na Cúria (Orvieto) .

1283 - 11 abril - Pagamento de um dote a Boécio de Dácia qui est in curia.

1283 - Bernier de Nivelles executor testamentário de um canone de Tongres.

1284 - 29 outubro - Pecham confirma a proibição de 18 março de 1277 .

1284 - 10 novembro - Carta de Pecham com alusão a morte de Siger.

1285 - 28 março - Morte de Martim IV.

1286 - 30 abril - Nova proibição em Oxford.

1286 - Bernier de Nivelles é indicado em Paris.

1299 a 1314 - Dante começa a Divina comédia.

1321 - 14 setembro - Morte de Dante. 